\title{
What do Social Determinants of Health Determine?
}

\author{
Judith Salerno • Kimber Bogard
}

Published online: 2 December 2019

(C) The New York Academy of Medicine 2019

\section{The Rise of SDOH}

Much has been written and discussed about the importance of the social determinants of health, specifically how social conditions can have a greater influence on health than the provision of health services. Social determinants of health have been described as the conditions in which people are born, grow, live, work, and age (World Health Organization, 2008).

There are a plethora of models and frameworks to elucidate key concepts of this idea to guide policy. A seminal report published in the Lancet put social determinants of health on the radar screen in the fields of health and medicine globally (Marmot, 2005). Since then, the phrase "social determinants of health" has become so common that it is often shortened to the acronym "SDOH." In that 2005 report, SDOH are described as the root causes of significant disparities in health outcomes between and within countries.

While the concept of SDOH is a vital one to understand and improve contemporary medicine and access to health, the terminology needs work. In 2019, it is clear that the phrase "social determinants of health" overstates the permanency of the relevant conditions and neglects the process of resilience and the assets which enable people to overcome social barriers to achieve good health outcomes.

J. Salerno $(\bowtie) \cdot$ K. Bogard

The New York Academy of Medicine, 1216 Fifth Avenue, New York, NY 10029, USA

e-mail: jsalerno@nyam.org

\section{Words Matter}

We can see all around us how much words matter. The words we use shape how we envision and implement solutions. Unfortunately, the term SDOH implies social conditions that are immutable in determining health outcomes, not merely influential in shaping them. Indeed, a determinant is defined as "an element that identifies or determines the nature of something or that fixes or conditions an outcome" (Merriam-Webster). But we know these conditions and outcomes are not fixed. "Determinants" not only sounds too determinative, but it negates any possibility for opportunity and change. In our work at the New York Academy of Medicine, with residents living in economically limited environments, we have learned about their resilience, tenacity, and hope that their families will be able to live a healthy life despite their context. Therefore, SDOH is not a term that resonates with what we are learning about people living in resource-constrained situations. Other organizations have similarly appreciated this distinction and we all recognize that merely changing our vocabulary is not a solution to addressing the root causes of inequities.

\section{SDOH 2.0}

The work highlighting the importance of $\mathrm{SDOH}$ was essential to turn policy attention from the role of individual behaviors in causing poor health outcomes to the social conditions that constrict opportunities to live a healthy life. Conditions such as healthy housing, reliable 
transportation, ample green space, and clean air, in addition to public safety, good paying jobs, quality education, and healthy food, are all important factors that influence health outcomes. These conditions influence, but do not determine health outcomes. We need better ways to capture what we know about the complex interplay among structural barriers that create social conditions associated with poor health outcomes, community assets, and the strength of people thriving against all odds. To find these, we need to first understand and acknowledge how these conditions came to be, then begin internal work to be a genuine partner, and engage people with lived experiences.

\section{1) Understand}

Huge divides persist between those with societal power and those without. Hundreds of years of structural racism, discrimination, entrenched exploitation and poverty, patriarchy, and marginalization have resulted in conditions that make living a healthy life more or less difficult depending on where you live, your social identity, and economic circumstance. Understanding the historical construction of social conditions that limit opportunity is necessary to be able to recognize and deconstruct existing barriers to live a healthy life.

\section{2) Work from Inside-Out}

Social conditions that influence health today in fact result from decades of disinvestment and marginalization by those in power, who have the privilege to make decisions. The New York Academy of Medicine has begun to look inwards and acknowledge our role in creating and perpetuating barriers that contribute to health inequities. We are undertaking an honest examination of the words we use to describe communities that face socially constructed barriers, acknowledging the complex interactions of personal, neighborhood, and structural factors that lead to health outcomes. Moving away from $\mathrm{SDOH}$, which implies immutable conditions, to a more accurate depiction of the multitude of factors that can be improved through interventions and, ultimately culture change, is imperative. Until institutions begin this journey internally, the solutions necessary to move beyond $\mathrm{SDOH}$ and achieve health equity will be out of reach.

\section{3) Engage}

We must foundationally change who is at the table to discuss and act on socially constructed conditions in order to dismantle the social factors that influence health and the words we use to describe them. Change can only happen when the people who bear the brunt of unjust policy decisions are at the table and empowered to have their voices heard and acted upon. When all voices are equally represented and heard, the opportunity will exist for open discussions about the actual lived experiences and effort it takes to overcome barriers. The ultimate goal in changing our thoughts, words, and actions together is to level the playing field by removing barriers that keep some people from having an equal shot at a healthy life.

At the New York Academy of Medicine, we do not have a "solution" and we are not ready to propose new language to describe one of the most important phenomena of contemporary scholarship in health and medicine. But we are ready to work harder to be more respectful and inclusive and to encourage our partners to do the same. I hope you will join us. 